\title{
Osteoclastogenesis inhibitor identified
}

The function of the transcription factor CCAAT/enhancer binding protein $\beta$ $(\mathrm{C} / \mathrm{EBP} \beta)$ in bone homeostasis is clearer following the discovery, by Achim Leutz's group, that $\mathrm{C} / \mathrm{EBP} \beta$ isoforms influence osteoclastogenesis by regulating the expression of the transcription factor MafB.

$\mathrm{C} / \mathrm{EBP} \beta$, which is involved in the differentiation and function of many cell types, exists as three different isoforms: two long transactivating forms $\left(\mathrm{LAP}^{\star}\right.$ and LAP) and a short repressive form (LIP), their successively truncated N-termini arising from alternative translation initiation. Leutz and colleagues compared the phenotypes of wild-type mice, C/EBP $\beta$ null $\left(c / e b p \beta^{-/-}\right)$mice and mice expressing only the LIP (truncated) isoform ( $L / L$ mice). Both types of genetically modified mouse displayed enhanced osteoclastogenesis, showing an increase in osteoclast size and bone resorption. "This similarity in outcome was difficult to understand at first," admits Leutz, "and implied the existence of an unknown inhibitor of osteoclastogenesis that is activated by long $\mathrm{C} / \mathrm{EBP} \beta$, the isoform that was lacking in both genotypes."

Gene-expression profiling identified this inhibitor as MafB. Treatment of wild-type osteoclast precursor cells with rapamycin, which inhibits the mammalian target of rapamycin (mTOR) pathway, induced MafB expression, enhanced LAP isoform production and inhibited osteoclastogenesis. Precursor cells from $c / e b p \beta^{-1-}$ or $L / L$ mice were largely unresponsive to rapamycin, underwent osteoclastogenesis and showed decreased MafB expression.

So, signaling through mTOR seems to influence $\mathrm{C} / \mathrm{EBP} \beta$ isoform levels, and, through MafB, osteoclastogenesis.

Katrin Legg

Original article Smink, J. et al. Transcription factor C/EBP $\beta$ isoform ratio regulates osteoclastogenesis through MafB. EMBO J. 28, 1769-1781 (2009). 